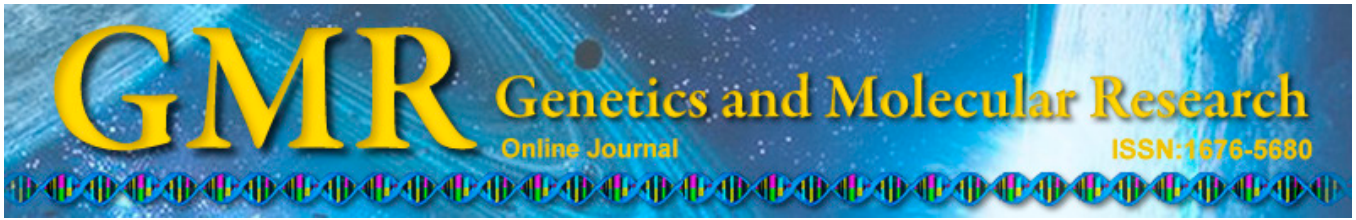

\title{
Consistency between molecular phylogeny and morphological classification of the Salix matsudana Koidz. complex (Salicaceae)
}

\author{
S.H. Du ${ }^{1,2 *}$, Z.S. Wang ${ }^{1,3 *}$, Y.X. Li ${ }^{1}$, D.S. Wang ${ }^{1}$ and J.G. Zhang ${ }^{1,3}$ \\ ${ }^{1}$ State Key Laboratory of Tree Genetics and Breeding, \\ Key Laboratory of Silviculture of the State Forestry Administration, \\ Research Institute of Forestry, Chinese Academy of Forestry, Beijing, China \\ ${ }^{2}$ College of Forestry, Shandong Agriculture University, Tai'an, Shandong, China \\ ${ }^{3}$ Collaborative Innovation Center of Sustainable Forestry in Southern China, \\ Nanjing Forestry University, Nanjing, Jiangsu, China \\ *Theses authors contributed equally to this study. \\ Corresponding author: J.G. Zhang \\ E-mail: zhangjg@caf.ac.cn
}

Genet. Mol. Res. 14 (3): 8663-8671 (2015)

Received October 15, 2014

Accepted March 12, 2015

Published July 31, 2015

DOI http://dx.doi.org/10.4238/2015.July.31.15

\begin{abstract}
The morphological species concept is based on morphological traits, which are often subject to subjectivity or artifact. Molecular evidence is needed to test the reliability of morphological classification of taxa that are controversial and to provide appropriate taxonomic delimitation. In this study, we used 15 single-copy nuclear loci and 2 chloroplast fragments to verify the morphological classification of the Salix matsudana Koidz. complex using phylogenetic approaches. Complete sequence alignment showed slight diversification in nuclear sequences and no variety in chloroplast DNA fragments. Phylogenetic trees revealed a monophyletic group consisting of all individuals of $S$. matsudana and 2 clades within this group, with a $100 \%$ bootstrap support value and 1.00 posterior probability. The topology of the phylogenetic trees was highly
\end{abstract}


consistent with the morphological classification of the S. matsudana complex. Verifying the genetic background of these classification units based on remarkable morphological differences will provide a foundation for future studies of Salix and the breeding of new horticultural varieties.

Key words: Morphology; Phylogeny; Salix matsudana; Species concept

\section{INTRODUCTION}

Species is the essential unit of concern in biodiversity, conservation, and other biological properties (Bradley and Baker, 2001). Various concepts of species have been developed (Fraser and Bernatchez, 2001). The morphological species concept is defined mainly based on the description of outlier taxonomic characters of different classification units. The phylogenetic species concept recognizes species as an irreducible cluster of individuals that is distinct from other clusters and within which there is an obvious pattern of ancestry and descent (Cracraft, 1989), or a species is a monophyletic group of common ancestry (de Queiroz and Gauthier, 1990). The development of molecular systematics over the past several decades has greatly advanced the phylogenetic species concept (Amato and Montresor, 2008). An inevitable aspect of morphological classification is the subjectivity or artifact, and thus molecular evidence is needed to test the reliability of morphological classification of taxa that are controversial (Su et al., 2012).

Salix matsudana Koidz. has 1 varietas and 3 forma, including S. matsudana var. pseudo-matsudana, S. matsudana f. pendula, S. matsudana f. tortuosa, and S. matsudana f. umbraculifera (Wu, 1999). Compared with S. matsudana var. matsudana, the annual branches of S. matsudana var. pseudo-matsudana, S. matsudana f. tortuosa, and S. matsudana f. pendula are long and drooped. The pistillate flower of $S$. matsudana var. pseudomatsudana contains 1 gland, while the other classification units contain 2. The branch of S. matsudana f. tortuosa is curly, which is distinctive in Salix. The tree form of S. matsudana f. umbraculifera is significantly different from other classification units, the crown of which is hemispherical and appears as a steamed bread. Because of the remarkably different morphological characters, it remains unclear whether these classification units should be treated as different species or as subspecies. Verifying the placement of these classification units and clarifying their evolutionary relationships will not only provide molecular evidence for the classification of controversial taxa but also set a foundation for future studies of functional genes related to horticultural traits. In this study, a phylogenetic approach based on the concordance of multiple single-copy orthologous nuclear loci and chloroplast sequences was utilized to testify the reliability of morphological classification and to evaluate the evolutionary relationships of different classification units in the S. matsudana complex.

\section{MATERIAL AND METHODS}

The biological material was sampled from the Beijing Botanical Garden and Summer Palace. Three to six individuals of each classification unit were sampled. Fresh leaves were 
collected and dried immediately with silica gel. Populus euphratica, Salix arbutifolia, Salix triandra, and Salix raddeana were used as outgroups in phylogenetic analysis. Details of the materials sampled are listed in Table 1.

\section{Table 1. Details of the materials sampled.}

\begin{tabular}{|c|c|c|c|c|}
\hline Samples & No. of individuals & Latitude & Longitude & Altitude (masl) \\
\hline S. matsudana var. matsudana & 5 & $39^{\circ} 59^{\prime} \mathrm{N}$ & $116^{\circ} 12^{\prime} \mathrm{E}$ & 73 \\
\hline S. matsudana var. pseudo-matsudana & 4 & $45^{\circ} 48^{\prime} \mathrm{N}$ & $126^{\circ} 31^{\prime} \mathrm{E}$ & 52 \\
\hline S. matsudana f. tortuosa & 5 & $39^{\circ} 59^{\prime} \mathrm{N}$ & $116^{\circ} 16^{\prime} \mathrm{E}$ & 67 \\
\hline S. matsudana f. pendula & 5 & & & \\
\hline S. matsudana f. umbraculifera & 5 & & & \\
\hline S. arbutifolia & 1 & $40^{\circ} 43^{\prime} \mathrm{N}$ & $124^{\circ} 47^{\prime} \mathrm{E}$ & 433 \\
\hline S. triandra & 1 & $47^{\circ} 22^{\prime} \mathrm{N}$ & $87^{\circ} 47^{\prime} \mathrm{E}$ & 20 \\
\hline S. raddeana & 1 & $40^{\circ} 41^{\prime} \mathrm{N}$ & $117^{\circ} 14^{\prime} \mathrm{E}$ & 410 \\
\hline P. euphratica & 1 & $47^{\circ} 41^{\prime} \mathrm{N}$ & $86^{\circ} 49^{\prime} \mathrm{E}$ & 670 \\
\hline
\end{tabular}

masl, meters above sea level.

\section{DNA extraction, polymerase chain reaction amplification, and sequencing}

The primers used for amplifying and sequencing single-copy nuclear loci in this study were developed in our previous study (Du et al., 2014). Primers for chloroplast DNA (cpDNA) $r b c l$ and $t r n K$ are listed in Table 2. All DNA sequences of this study have been submitted to GenBank (\#KJ101449-KJ101504 and \#KJ155027-KJ155446).

Table 2. Primers used for $r b c l$ and $t r n K$ in this study (primers underlined are internal sequencing primers).

\begin{tabular}{|c|c|c|c|c|}
\hline Locus & Primer & Sequence $\left(5^{\prime}-3^{\prime}\right)$ & $\mathrm{Ta}\left({ }^{\circ} \mathrm{C}\right)$ & References \\
\hline \multirow[t]{4}{*}{$\operatorname{trn} K$} & $\operatorname{trnKF}$ & GGGTTGCCCGGGACTCGAAC & 58 & modified from Demesure et al. (1995) \\
\hline & $\operatorname{trnkR}$ & ATTGGATTTGCTGTGATA & & \\
\hline & $\operatorname{trnK}-3 \mathrm{~F}$ & ACTAATGGGATGTCCTACTG & & Wang et al. (2014) \\
\hline & $\operatorname{trnK}-3 \mathrm{R}$ & GATTTCTAGTCACCTATTAC & & \\
\hline \multirow[t]{2}{*}{ rbcl } & $\overline{\mathrm{rbclF}}$ & ATGTCACCACAAACAGAAACT & 56 & Bobowski et al. (1999) \\
\hline & rbclR & CTTCACAAGCAGCAGCTAGTTCAGGACTCC & & \\
\hline
\end{tabular}

Total genomic DNA was extracted from $30 \mathrm{mg}$ leaf tissue from each individual using a modified protocol that has been described previously (Doyle, 1987). Polymerase chain reaction was performed in a total volume of $30 \mu \mathrm{L}$ containing 10-60 ng genomic DNA, $0.8 \mu \mathrm{M}$ of each dNTP, $2.4 \mu \mathrm{M}$ of each primer, $0.15 \mathrm{U}$ exTaq DNA polymerase (TaKaRa, Shiga, Japan), and $2.0 \mathrm{mM} \mathrm{MgCl}$. Amplification was carried out in a temperature gradient $96 \mathrm{U}$ thermocycler (Applied Biosystems, Foster City, CA, USA) as follows: 5 min at $94^{\circ} \mathrm{C}$, followed by 35 cycles of $30 \mathrm{~s}$ at $94^{\circ} \mathrm{C}, 60 \mathrm{~s}$ at $50-60^{\circ} \mathrm{C}$ (depending on the annealing temperature of specific primers and length of the amplified regions), $90 \mathrm{~s}$ at $72^{\circ} \mathrm{C}$, and a final extension at $72^{\circ} \mathrm{C}$ for 10 min. Products were examined by agarose gel electrophoresis and purified using a DNA Purification kit (Amersham Pharmacia Biotech, Piscataway, NJ, USA). The purified DNA was directly sequenced using an ABI 3730XL DNA analyzer (Applied Biosystems). The same primers were utilized for amplification and sequencing. 


\section{Data analysis}

The assembled contigs of each individual were aligned using CLUSTAL X (Thompson et al., 1997) and refined manually in BioEdit (Hall, 1999). Homogeneity across nuclear DNA was tested using the incongruence length difference test (Farris et al., 1994) as implemented in PAUP* 4.0b10* (Swofford, 2003). A heuristic search was performed with 10 random addition replicates and tree-bisection-reconnection branch swapping. Phylogenetic trees were reconstructed using maximum parsimony (MP) and Bayesian methods. MP analysis was conducted in PAUP* 4.0b10* (Swofford, 2003), with all characters equally weighted and treated as unordered and gaps treated as missing data. A heuristic search was performed with tree-bisection-reconnection branch swapping, MULPARS option, and RANDOM stepwise addition with 1000 replicates. Topological robustness was assessed by bootstrap analysis with 1000 replicates using simple taxon addition (Felsenstein, 1985). An appropriate nucleotide substitution model for each locus was determined using Modeltest 3.7 (Posada, 2003). Models were chosen mainly based on the Akaike information criterion and used for subsequent Bayesian analysis. Bayesian analysis was conducted using Mrbayes 3.2.1 (Ronquist and Huelsenbeck, 2003). Two independent runs of Metropolis-coupled Markov chain Monte Carlo were conducted simultaneously, with each run including 1 cold chain and 3 incrementally heated chains, starting randomly in the parameter space. A total of 2,000,000 generations were run, and trees were sampled once every 100 generations. The Tracer v1.5 program (Drummond and Rambaut, 2007) was utilized to evaluate stationary characteristics. The first $25 \%$ of sampled trees were discarded as burn-ins, and the posterior probabilities were calculated from the remaining trees. The phylogenetic trees were viewed in FigTree v 1.3.1 (Rambaut, 2008).

\section{RESULTS}

\section{Sequence characteristics}

We successfully obtained all 15 nuclear DNA sequences and 2 cpDNA fragments for all individuals. The characteristics of the 17 loci and their most appropriate nucleotide substitution models are summarized in Table 3. After removing regions showing ambiguous alignment, the aligned length of the nuclear loci ranged from 329 to $1054 \mathrm{bp}$ with a total length of $8599 \mathrm{bp}$. The number of variable and informative sites ranged from 1-34 and 1-32, with total numbers of 218 and 203, respectively. The sequence length of $r b c l$ ranged from 978 to $1329 \mathrm{bp}$, with an aligned length of $924 \mathrm{bp}$. The aligned length of $\operatorname{trnK}$ was $2440 \mathrm{bp}$, and the sequencing length varied from 2445 to $2562 \mathrm{bp}$, in which there was an indel of 21 bp. Unexpectedly, no variable sites were detected in the $r b c l$ and $t r n K$ sequences of all 24 individuals of $S$. matsudana. To confirm this, we repeated the amplification and sequencing for all individuals and observed the same result.

\section{Phylogenetic analysis of the nuclear loci}

Phylogenetic trees generated based on individual nuclear DNA sequences were 
Table 3. Characteristics of nuclear and cpDNA loci and the combined data (excluding outgroups).

\begin{tabular}{lrrrll}
\hline $\begin{array}{l}\text { Locus } \\
\text { (without outgroups) }\end{array}$ & $\begin{array}{c}\text { Aligned } \\
\text { length }\end{array}$ & $\begin{array}{c}\text { No. of variable } \\
\text { sites (\%) }\end{array}$ & $\begin{array}{r}\text { No. of informative } \\
\text { sites (\%) }\end{array}$ & $\begin{array}{l}\text { Nucleotide } \\
\text { substitution model }\end{array}$ & $\begin{array}{l}\text { Gamma shape } \\
\text { parameter }\end{array}$ \\
\hline Nuclear DNA & & & & & \\
DSL 1 & 426 & $25(5.87)$ & $24(5.63)$ & HKY+I+G & 0.2473 \\
DSL 2 & 329 & $3(0.91)$ & $2(0.61)$ & F81 & equal \\
DSL 3 & 540 & $24(4.44)$ & $24(4.44)$ & JC & equal \\
DSL 4 & 427 & $4(0.94)$ & $4(0.94)$ & JC & equal \\
DSL 5 & 403 & $6(1.49)$ & $6(1.49)$ & F81 & equal \\
DSL 6 & 473 & $12(2.54)$ & $12(2.54)$ & F81 & equal \\
DSL 7 & 390 & $10(2.56)$ & $9(2.31)$ & HKY & equal \\
DSL 8 & 476 & $1(0.21)$ & $1(0.21)$ & F81 & equal \\
DSL 24 & 1054 & $25(2.37)$ & $22(2.09)$ & F81+I+G & 0.6230 \\
DSL 25 & 635 & $10(1.57)$ & $9(1.42)$ & F81 & equal \\
DSL 26 & 504 & $2(0.40)$ & $1(0.20)$ & JC & equal \\
DSL 28 & 825 & $28(3.39)$ & $27(3.27)$ & F81+I+G & 1.1356 \\
DSL 29 & 834 & $34(4.07)$ & $32(3.84)$ & TrN+I+G & 0.8756 \\
DSL 33 & 531 & $23(4.33)$ & $21(3.95)$ & F81+I+G & 0.5468 \\
DSL 35 & 752 & $11(1.46)$ & $9(1.20)$ & F81 & equal \\
Concatenated data & 8599 & $218(2.54)$ & $203(2.36)$ & HKY+I+G & 0.6165 \\
cpDNA & & & & & \\
rbcl & 924 & 0 & 0 & & \\
trnK & 2440 & 0 & 0 & & \\
\hline
\end{tabular}

very similar to each other (results not shown). The incongruence length difference test showed significant incongruence among the nuclear DNA sequences $(P=0.03)$. However, previous studies have examined the limitations of this statistical test (Hipp et al., 2004; Quicke et al., 2007). Therefore, we combined the 15 individual nuclear DNA loci into a single data set to sufficiently reconstruct the phylogeny of classification units in S. matsudana.

The combined data set generated the 121 most parsimonious trees of 837 steps with a consistency index of 0.81 and a retention index of 0.71 using the MP method, which recovered a fully resolved monophyletic group consisting of all 24 individuals with a $100 \%$ bootstrap support value (Figure 1). This group was also yielded through Bayesian analysis with a 1.00 posterior probability (Figure 2). Within the monophyletic group, all individuals were subdivided into 2 clades (I and II) with 100\% bootstrap support and 1.00 posterior probability. Individuals of $S$. matsudana var. pseudo-matsudana clustered in clade I, while the sister clade, clade II, comprised the remaining individuals. Within this clade, individuals of different classification units were scattered and most braches were polytomic. In the Bayesian phylogenetic tree, several branches with relatively high posterior probability were recovered. However, not all individuals of the same classification unit were clustered together in 1 branch or individuals from different classification units linked to each other in 1 clade. For example, 3 individuals of $S$. matsudana f. pendula grouped into 1 clade, but the remaining samples clustered with $S$. matsudana f. umbraculifera and S. matsudana var. matsudana in another clade. Furthermore, these branches were not supported or showed relatively low bootstrap support values in the MP tree.

\section{DISCUSSION}

With a large data set of combined 15 single-copy nuclear loci and 2 cpDNA fragments, the phylogeny of the S. matsudana complex was reconstructed (Figures 1 and 2). All 


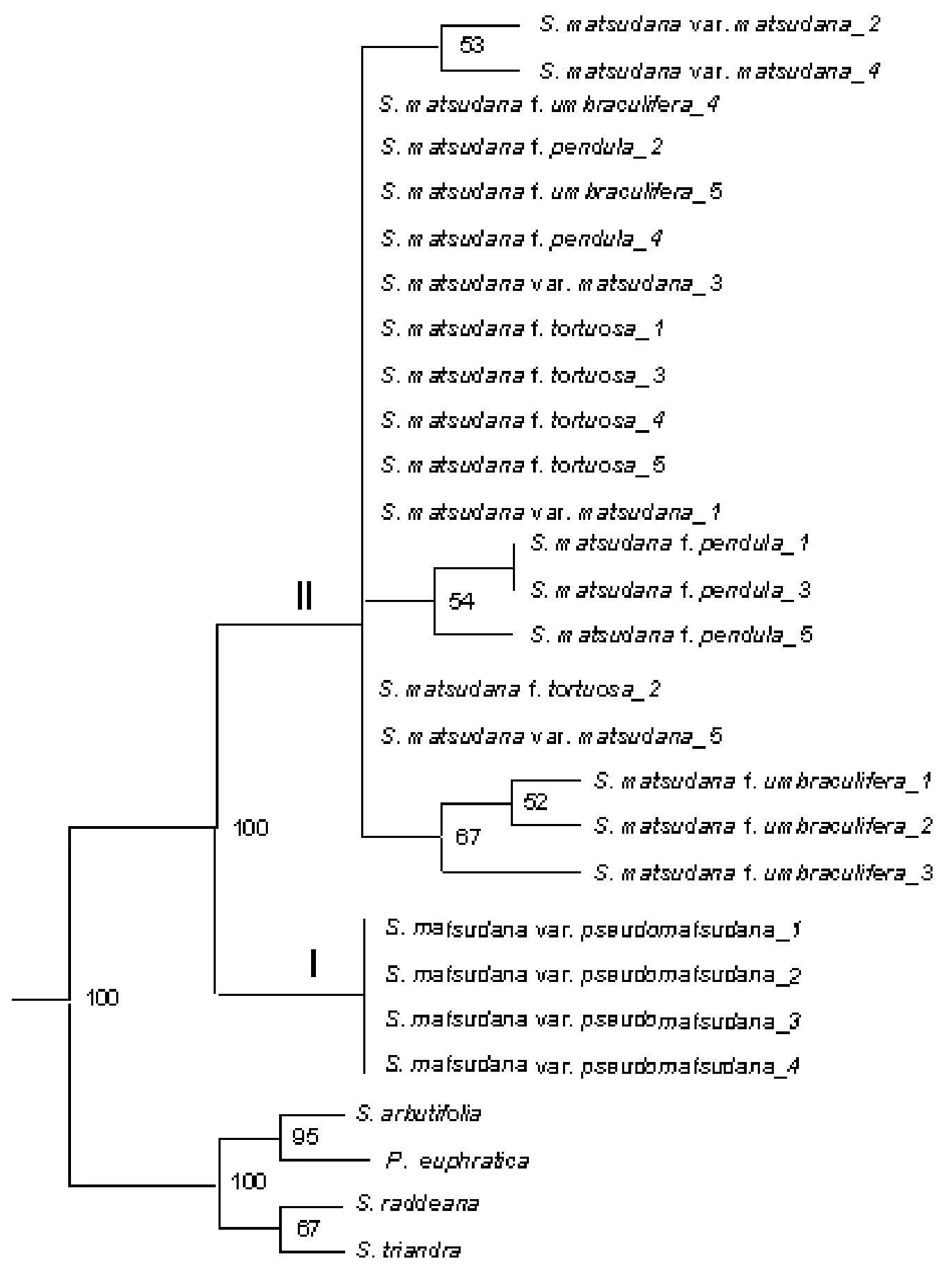

Figure 1. Phylogenetic tree reconstructed based on the MP method. Numbers after scientific name indicate the individual order. Bootstrap support values are listed next to nodes. 


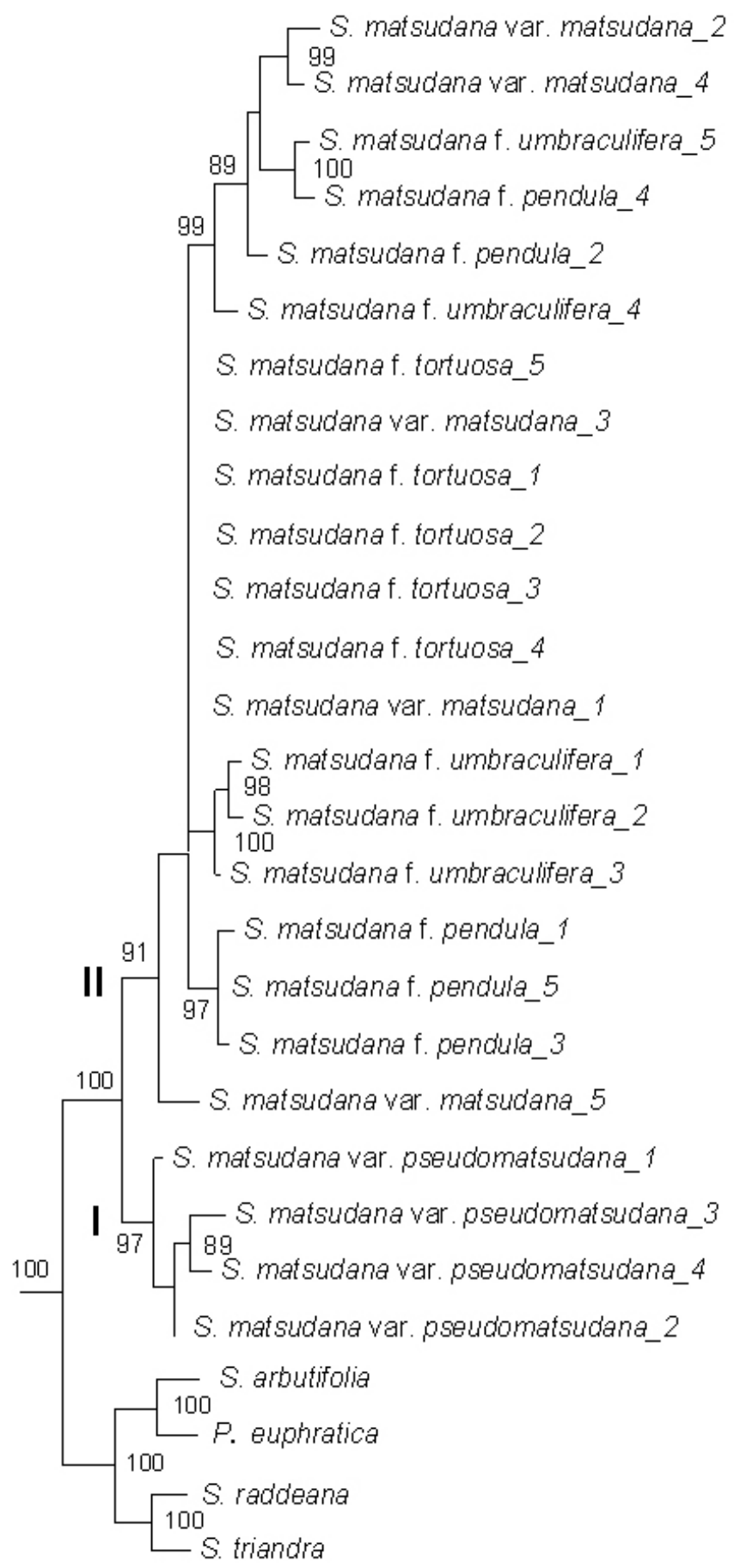

Figure 2. Phylogenetic tree reconstructed based on the Bayesian method. Numbers after scientific names indicate the individual order. Posterior probabilities are listed next to the nodes. 
individuals of $S$. matsudana grouped together as a monophyletic group separate from the outgroup species with $100 \%$ bootstrap support value or 1.00 posterior probability, revealing the close relationships between classification units.

In the 2 phylogenetic trees, individuals of S. matsudana var. pseudo-matsudana formed a single clade sister to other remaining individuals with $100 \%$ bootstrap and 1.00 posterior probability (Figures 1 and 2). Furthermore, S. matsudana var. pseudo-matsudana showed significant morphological differences from other classification units as the only varietas in the S. matsudana complex. The female flower has only 1 gland, while the others have $2(\mathrm{Wu}, 1999)$. Based on the nuclear phylogenetic trees and no variable sites in cpDNA fragments, we support the traditional classification of S. matsudana var. pseudo-matsudana as a varietas in S. matsudana.

The remaining classification units showed significant morphological differences; however, slight differences were detected at the molecular level (clade II in Figures 1 and 2). Furthermore, no variable sites existed in cpDNA fragments. Although some nodes were supported by high posterior probability $(>95 \%)$ in the Bayesian phylogenetic tree, it is wellknown that posterior probability calculated from actual data for some clades often appears to be too high (Yang and Rannala, 2012). Furthermore, internal nodes within clade II were supported by a low bootstrap support value $(\sim 50 \%)$ in the MP tree. Overall, the classification units were not sufficiently diversified at the molecular level and thus were classified as different forma with close evolutionary relationships.

The number of variable sites in the 15 single-copy nuclear loci of the 24 individuals was relatively high; however, extremely weak resolution in the phylogenetic trees showed that most of the variation was commonly shared by these classification units. Furthermore, there were no variable sites in the cpDNA $r b c l$ and $\operatorname{trn} K$ sequences, which have been widely utilized in phylogenetic studies at the species or genus level (Kusumi et al., 2000; Soltis et al., 2001; Fan and Xiang, 2003; Guo and Ge, 2005; Tang et al., 2010). Shared variation in nuclear loci and no variation in cpDNA sequences of the classification units indicated that these classification units were not sufficiently diverse to be treated as different taxonomic species and that they belonged to 1 species, $S$. matsudana, both according to the morphological and phylogenetic species concept. The conspicuous morphological differences among the varietas and forma of S. matsudana may result from the differential expression of a small number of genes or a unique gene. Determining the genetic background of the morphological differences will set the stage for a broad range of future studies in S. matsudana and the breeding of new horticultural varieties.

\section{ACKNOWLEDGMENTS}

We thank Dr. Zou Xinhui and Dr. Tang Liang for phylogenetic analysis. We also thank Dr. Zeng Yanfei for data analysis and suggestions on a preliminary version of this paper. Research supported by the Specialized Research Fund for Young Scholars of the Research Institute of Forestry, Chinese Academy of Forestry (\#RIF 2012-06).

\section{REFERENCES}

Amato A and Montresor M (2008). Morphology, phylogeny, and sexual cycle of Pseudo-nitzschia mannii sp. nov. (Bacillariophyceae): a pseudo-cryptic species within the P. pseudodelicatissima complex. Phycologia 47: 487-497. Bobowski B, Hole D, Wolf P, Bryant L, et al. (1999). Identification of roots of woody species using polymerase chain 
reaction (PCR) and restriction fragment length polymorphism (RFLP) analysis. Mol. Ecol. 8: 485-491.

Bradley RD and Baker RJ (2001). A test of the genetic species concept: cytochrome-b sequences and mammals. $J$. Mammal. 82: 960-973.

Cracraft J (1989). Speciation and its ontology: the empirical consequences of alternative species concepts for understanding patterns and processes of differentiation. In: Speciation and its Consequences (Otte D and Endler JA, eds.). Sinauer, Sunderland, 28-59.

de Queiroz K and Gauthier J (1990). Phylogeny as a central principle in taxonomy: phylogenetic definitions of taxon names. Syst. Biol. 39: 307-322.

Demesure B, Sodzi N and Petit R (1995). A set of universal primers for amplification of polymorphic non-coding regions of mitochondrial and chloroplast DNA in plants. Mol. Ecol. 4: 129-134.

Doyle JJ (1987). A rapid DNA isolation procedure for small quantities of fresh leaf tissue. Phytochem. Bull. 19: 11-15.

Drummond AJ and Rambaut A (2007). BEAST: Bayesian evolutionary analysis by sampling trees. BMC Evol. Biol. 7 : 214.

Du SH, Wang ZS and Zhang JG (2014). A novel set of 15 single-copy nuclear DNA markers for genetic studies of Salicaceae. Genet. Mol. Res. 13: 4911-4917.

Fan C and Xiang QYJ (2003). Phylogenetic analyses of Cornales based on 26S rRNA and combined 26S rDNA-matKrbcL sequence data. Am. J. Bot. 90: 1357-1372.

Farris JS, Källersjö M, Kluge AG and Bult C (1994). Testing significance of incongruence. Cladistics 10: 315-319.

Felsenstein J (1985). Confidence limits on phylogenies: an approach using the bootstrap. Evolution 39: 783-791.

Fraser DJ and Bernatchez L (2001). Adaptive evolutionary conservation: towards a unified concept for defining conservation units. Mol. Ecol. 10: 2741-2752.

Guo YL and Ge S (2005). Molecular phylogeny of Oryzeae (Poaceae) based on DNA sequences from chloroplast, mitochondrial, and nuclear genomes. Am. J. Bot. 92: 1548-1558.

Hall TA (1999). BioEdit: a user-friendly biological sequence alignment editor and analysis program for Windows 95/98/ NT. Nucleic Acids Symp. Ser. 41: 95-98.

Hipp AL, Hall JC and Sytsma KJ (2004). Congruence versus phylogenetic accuracy: revisiting the incongruence length difference test. Syst. Biol. 53: 81-89.

Kusumi J, Tsumura Y, Yoshimaru H and Tachida H (2000). Phylogenetic relationships in Taxodiaceae and Cupressaceae sensu stricto based on matK gene, chlL gene, trnL-trnF IGS region, and trnL intron sequences. Am. J. Bot. 87: 14801488.

Posada D (2003). Using Modeltest and PAUP* to select a model of nucleotide substitution. Curr. Protoc. Bioinformatics 6.5. 1-6.5. 14.

Quicke DL, Jones OR and Epstein DR (2007). Correcting the problem of false incongruence due to noise imbalance in the incongruence length difference (ILD) test. Syst. Biol. 56: 496-503.

Rambaut A (2008). FigTree v1. 1.1: Tree figure drawing tool. Available at [http://ree.bio.ed.ac.uk/software/figtree/]. Accessed October 30, 2006.

Ronquist F and Huelsenbeck JP (2003). MrBayes 3: Bayesian phylogenetic inference under mixed models. Bioinformatics 19: 1572-1574.

Soltis DE, Tago-Nakazawa M, Xiang QY, Kawano S, et al. (2001). Phylogenetic relationships and evolution in Chrysosplenium (Saxifragaceae) based on matK sequence data. Am. J. Bot. 88: 883-893.

Su JX, Wang W, Zhang LB and Chen ZD (2012). Phylogenetic placement of two enigmatic genera, Borthwickia and Stixis, based on molecular and pollen data, and the description of a new family of Brassicales, Borthwickiaceae. Taxon 61: 601-611.

Swofford DL (2003). PAUP*: phylogenetic analysis using parsimony (*and other methods), Sinauer Associates, Sunderland.

Tang L, Zou X, Achoundong G, Potgieter C, et al. (2010). Phylogeny and biogeography of the rice tribe (Oryzeae): evidence from combined analysis of 20 chloroplast fragments. Mol. Phylogenet. Evol. 54: 266-277.

Thompson JD, Gibson TJ, Plewniak F, Jeanmougin F, et al. (1997). The CLUSTAL_X windows interface: flexible strategies for multiple sequence alignment aided by quality analysis tools. Nucleic Acids Res. 25: 4876-4882.

Wang Z, Du S, Selvadurai D, Wang D, et al. (2014). Phylogeny reconstruction and hybrid analysis of Populus (Salicaceae) based on nucleotide sequences of multiple single-copy nuclear genes and plastid fragments. PLoS One 9: e103645. doi: 10.1371/journal.pone.0103645.

Wu Z (1999). Flora of China. Science Press, Beijing.

Yang Z and Rannala B (2012). Molecular phylogenetics: principles and practice. Nat. Rev. Genet. 13: 303-314. 